Reprod. Nutr. Dévelop., 1988, 28 Suppl. $\mathrm{n}^{\circ} 1,179-180$

\title{
Influence d'injections biquotidiennes de 1-29 GRF sur la production, l'ingestion et l'état nutritionnel de la chèvre en lactation
}

D. SAUVANT, G. KANN $\left({ }^{*}\right)$, J. HERVIEU, Catherine DISENHAUS

Station de Nutrition et Alimentation (I.N.R.A.) de II.N.A.-P.G.

16. rue Claude-Bernard. 75231 Paris Cedex 05.

(*) Laboratoire de Physiologie et de la Lactation (I.N.R.A.),

78350 Jouy-en-Josas.

Summary. Daily injections of GRF 1-29 increased significantly milk production of goats. Effects of injections were slightly positive on voluntary intake and negative on energy balance.

Les effets galactogène du GRF 1-29 ont déjà été étudiés sur des vaches (Enright et al. ; Kann et Périer, non publié) et des brebis (Kann et Périer, non publié). Le travail présenté a pour objet de confirmer cet effet chez la chèvre.

Matériel et méthodes. Vingt-huit chèvres, Alpines ou Saanen, placées en cages individuelles, ont reçu un même régime alimentaire constitué de foin de luzerne offert en quantité limitée $(0,7$ à $1,0 \mathrm{~kg}$ de matière sèche (MS) selon le niveau de production (NP), de pulpes de betteraves surpressées ensilées distribuées ad libitum (0,4 à $1,1 \mathrm{~kg}$ de MS ingérée selon le NP) et d'un aliment concentré composé distribué à raison de $0,35 \mathrm{~kg} / \mathrm{kg}$ de lait à $3,5 \%$ de taux butyreux au-dessus d'un niveau de production de $0,7 \mathrm{~kg} / \mathrm{jour}$. L'ajustement de l'apport de concentré a été effectué toutes les deux semaines selon un programme préalablement établi.

Après une pré-période de 3 semaines, les animaux, qui étaient dans leur $3^{\mathrm{e}}$ mois de lactation, ont été classés en 14 blocs de niveau de production pour constituer 2 lots. Les chèvres du lot traité (G) ont reçu une injection biquotidienne de GRF 1-29 Sanofi Recherches (Montpellier) à la dose 0,76 nanomole/kg par voie intraveineuse, les animaux témoins du lot témoin ( $T$ ) n'ont pas reçu de placebo. Un échantillon individuel de sang a été prélevé une semaine sur deux en vue du dosage du glucose, des acides gras non estérifiés, du $\beta-\mathrm{OH}$ butyrate et de l'urée. La période expérimentale a été de 8 semaines. Pour préciser l'évolution des effets, les analyses de variance ont été effectuées sur les résultats moyens de 4 périodes successives (1,2,3 et 4 ) de 2 semaines en prenant comme co-variable la mesure de la même caractéristique à la pré-période.

Résultats. Après chaque administration de GRF 1-29, la GH est déchargée pendant au moins $90 \mathrm{~min}$. Dans toutes les analyses, la co-variable a eu une influence significative à $P<0,05$ ou $P<0,01$. Les niveaux d'ingestion de matière sèche totale ont été non significativement supérieurs pour le lot $G$; l'écart absolu ou relatif entre les 2 lots n'a cessé de croître dans le temps (tabl. 1) en raison de différences enregistrées sur la pulpe et le foin. En période 1, les quantités produites de lait, de matières grasses et de protéines ont été supérieures pour le lot traité $\mathrm{G}$ sans que la différence soit significative. Par contre, un écart significatif a été enregistré pour la production de lait et de protéines aux périodes 2,3 et 4 
(tabl. 1). L'effet progressif du GRF s'est traduit par la persistance significativement meilleure chez les animaux du lot G. ( -10 contre $-65 \mathrm{~g}$ de lait/semaine). Le gain absolu de production de lait brut obtenu par le GRF ne diffère pas statistiquement selon le niveau de production des animaux. Pour la production de matières grasses, l'écart G-T n'a été significatif qu'en $3^{\mathrm{e}}$ période, la différence étant surtout expliquée par les acides gras longs du lait, donc peut être par le niveau de lipomobilisation. II n'y a pas de différence significative pour les taux butyreux et protéique du lait.

TABL. 1. - Evolution des principales caractéristiques zootechniques pendant les 4 périodes de mesures.

\begin{tabular}{|c|c|c|c|c|c|c|c|}
\hline & & & \multicolumn{4}{|c|}{ Périodes } & \multirow[b]{2}{*}{ Moyenne } \\
\hline & & & 1 & 2 & 3 & 4 & \\
\hline Matière sèche ingérée & $(g / j)$ & $\begin{array}{l}\mathrm{m} \\
\mathrm{d}\end{array}$ & $\begin{array}{r}2397 \\
43\end{array}$ & $\begin{array}{r}2433 \\
49\end{array}$ & $\begin{array}{r}2303 \\
59\end{array}$ & $\begin{array}{r}2288 \\
71\end{array}$ & $\begin{array}{r}2355 \\
56\end{array}$ \\
\hline Lait & $(g / j)$ & $\begin{array}{l}\mathrm{m} \\
\mathrm{d}\end{array}$ & $\begin{array}{r}3647 \\
48\end{array}$ & $\begin{array}{r}3441 \\
247^{*}\end{array}$ & $\begin{array}{r}3313 \\
468^{*}\end{array}$ & $\begin{array}{r}3164 \\
377^{*}\end{array}$ & $\begin{array}{r}3391 \\
285^{*}\end{array}$ \\
\hline Matières grasses & $(g / j)$ & $\begin{array}{l}\mathrm{m} \\
\mathrm{d}\end{array}$ & $\begin{array}{r}97,5 \\
0,8\end{array}$ & $\begin{array}{r}94,4 \\
6,4\end{array}$ & $\begin{array}{c}80,1 \\
9,9^{*}\end{array}$ & $\begin{array}{r}83,6 \\
8,5\end{array}$ & $\begin{array}{c}88,9 \\
6,4^{*}\end{array}$ \\
\hline Matières protéiques & $(g / j)$ & $\mathrm{m}$ & $\begin{array}{r}93,0 \\
7,0\end{array}$ & $\begin{array}{c}92,2 \\
7,9^{*}\end{array}$ & $\begin{array}{l}81,9 \\
12,3^{* *}\end{array}$ & $\begin{array}{l}85,9 \\
13,2^{* *}\end{array}$ & $\begin{array}{l}88,2 \\
10,1^{\text {** }}\end{array}$ \\
\hline $\begin{array}{l}\text { Différence de bilans } \\
\text { - Energie } \\
\text { - PDI }\end{array}$ & $\begin{array}{l}(\mathrm{G}-\mathrm{T}) \\
\text { UFL/j } \\
\mathrm{gPDI} / \mathrm{j}\end{array}$ & $\begin{array}{l}d \\
d\end{array}$ & $\begin{array}{l}0,04 \\
6,50\end{array}$ & $\begin{array}{r}-0,04 \\
0,60\end{array}$ & $\begin{array}{l}-0,11 \\
-6,00\end{array}$ & $\begin{array}{r}-0,10 \\
-10,00\end{array}$ & \\
\hline
\end{tabular}

$\overline{\mathrm{m}}=$ Moyenne des animaux du lot témoin $(T) ; \quad \mathrm{d}=$ Différence entre les lots $\mathrm{G}$ et $\mathrm{T}$; $\quad$ * = Significatif à $\mathrm{P}<0,05 ;{ }^{* *}=$ Significatif à $\mathrm{P}<0,05$.

Les bilans énergétique et azoté calculés n'ont pas été différents entre les 2 lots ; cependant, l'écart G-T a évolué en défaveur du lot G. Les gains de poids vif ont été assez importants pour cette période de la lactation et non différents entre les 2 lots ( 445 et $527 \mathrm{~g} /$ semaine pour les lots T et G). Des différences G-T significatives de glycémie de 44 et $45 \mathrm{mg} / \mathrm{l}$ ont été observées en période 1 et 4 respectivement. En période 1, les chèvres du lot témoin ont eu une teneur en AGNE significativement supérieure de $167 \mathrm{eq} / \mathrm{l}$; cet écart, difficilement explicable, a eu tendance à s'inverser aux périodes suivantes $(2,9$ et $31 \mu \mathrm{E} / \mathrm{I})$. L'urémie du lot $\mathrm{G}$ a été inférieure de 62,61, 64 et $92 \mathrm{mg} / \mathrm{l}$; ces différences, qui pourraient traduire une meilleure utilisation de l'azote sous l'influence du GRF, ont été à un niveau de tendance $(P<0,10)$ aux périodes $1,2,3$, et significatif $(P<0,01)$ en période 4. Aucun écart significatif n'a pu être décelé dans les teneurs en $\beta-\mathrm{OH}$-butyrate du plasma et I'hématocrite.

En conc/usion, les injections répétées de GRF 1-29 ont significativement favorisé les productions mammaires et la persistance de la lactation. La dépense accrue de production n'a pas été compensée par le léger accroissement de l'ingestion; néanmoins, le dispositif expérimental n'a pas permis de mettre en évidence une différence d'intensité de reconstitution des réserves corporelles entre les 2 lots.

Enright W. J., Chapin W. H., Moseley W. M., Zinn S. A., Allentucker H., 1986. J. Dairy Sci., 69, 344-351. 